\title{
Quantifying model uncertainty in dynamical systems driven by non-Gaussian Lévy stable noise with observations on mean exit time or escape probability
}

\author{
Ting Gao and Jinqiao Duan \\ Department of Applied Mathematics, Illinois Institute of Technology \\ Chicago, IL 60616, USA \\ E-mail:tinggao0716@gmail.com,duan@iit.edu
}

February 24, 2016

\begin{abstract}
Complex systems are sometimes subject to non-Gaussian $\alpha$-stable Lévy fluctuations. A new method is devised to estimate the uncertain parameter $\alpha$ and other system parameters, using observations on mean exit time or escape probability for the system evolution. It is based on solving an inverse problem for a deterministic, nonlocal partial differential equation via numerical optimization. The existing methods for estimating parameters require observations on system state sample paths for long time periods or probability densities at large spatial ranges. The method proposed here, instead, requires observations on mean exit time or escape probability only for an arbitrarily small spatial domain. This new method is beneficial to systems for which mean exit time or escape probability is feasible to observe.

Key Words: Stochastic dynamical systems; parameter estimation; optimization; non-Gaussian Lévy motion; mean exit time; doublewell system

Mathematics Subject Classifications (2010): 65N21, 60H15, $60 \mathrm{~F} 10$
\end{abstract}

\section{Introduction}

Random fluctuations in complex systems are sometimes non-Gaussian $\alpha$-stable Lévy motions [35, 33, 34]. We consider a system under such fluctuations 
modeled by a scalar stochastic differential equation (SDE)

$$
\mathrm{d} X_{t}=f\left(\beta, X_{t}\right) \mathrm{d} t+\mathrm{d} L_{t}^{\alpha}, \quad X_{0}=x,
$$

where $X_{t}$ is the system state process, $f$ is a vector field (or drift), $\beta$ is a system parameter, and $L_{t}^{\alpha}$ is a scalar symmetric $\alpha$-stable Lévy motion $(0<\alpha<2)$ defined in a probability space $(\Omega, \mathcal{F}, \mathbb{P})$. For example, the calcium signal, as a proxy for climate state, in paleoclimatic data is approximately described [9] by a model like (11).

An $\alpha$-stable Lévy motion is a non-Gaussian process, while the wellknown Brownian motion is a Gaussian process. Non-Gaussian dynamical systems like (1) have attracted considerable attention recently [2], as they are appropriate models for various systems under heavy tail fluctuations [1, 30].

The process $L_{t}^{\alpha}$ has heavy tail or power law distribution in the sense that

$$
\mathbb{P}\left(\left|L_{t}^{\alpha}\right|>x\right) \sim \frac{1}{x^{\alpha}}
$$

for large $x$. The $\alpha$ is called the power parameter, or stability index, or nonGaussianity index. (In fact, Brownian motion corresponds to the special case $\alpha=2$. But, we will not consider this case in the present work.)

The $\alpha$-stable fluctuations arise in various situations, including modeling for optimal foraging, human mobility and geographical spreading of emergent infectious disease. GPS data are used to track the wandering black bowed albatrosses around an island in the Southern Indian Ocean to study their movement patterns in searching for food. The movement patterns obey a power law distribution [18] with power parameter $\alpha \approx 1.25$. One way to examine the human mobility is to collect data by online bill trackers, which provide successive spatial-temporal trajectories. A bill traveling at certain distances within a short period of time (less than one week) follows a power law distribution [6] with power parameter $\alpha \approx 1.6$. Moreover, the spreading patterns of human influenza, as described by the classic susceptiblenessinfection-recovery (SIS) epidemiologic model, is also strikingly similar to an $\alpha$-stable Lévy motion.

To make (11) a predictive model, it is essential to estimate the parameter $\alpha$, using observations on the system evolution. Methods for estimating the other system parameter $\beta$, when $\alpha$ is known, have been considered in literature ([16, 17, 36, e.g.]) and thus it is not a focus here. There are a couple of attempts in estimating $\alpha$. For example, assuming the drift ' $f$ ' insignificant (which is an inappropriate assumption in many situations), it is suggested [9, 35] to roughly estimate this $\alpha$ value using data on probability density function for $X_{t}$. The tail of the probability density function $p(x)$ behaves like $1 / x^{\alpha}$ for $x \gg 1$, after ignoring the drift ' $f$ '. Thus the $\log p$ vs. $\log x$ 
plot is a straight line with slope ' $-\alpha$ '. This provides an estimate $\alpha$ by data fitting. This method is not accurate as it assumes that the drift $f$ does not alter the tail behavior of $X_{t}$. Another approach to estimate $\alpha$ is suggested in [23] and it requires observations on lots of system state sample paths or sample characteristic functions for long time periods.

In the present paper, we devise a method to estimate $\alpha$ (and other system parameters), using observations on mean exit time or escape probability. Recall the first exit time of $X_{t}$ starting at $x$ (or 'a particle starting at $x$ ') from a bounded domain $D$ is defined as

$$
\tau(\omega):=\inf \left\{t \geq 0, X_{t}(\omega, x) \notin D\right\}
$$

and the mean exit time (also called mean residence time) is denoted as $u(x):=\mathbb{E} \tau$. The likelihood of a particle, starting at a point $x$, first escapes from a domain $D$ and lands in a subset $E$ of $D^{c}$ (the complement of $D)$ is called escape probability and is denoted by $P_{E}(x)$.

Both the mean exit time $u(x)$ and escape probability $P_{E}(x)$ satisfy deterministic, nonlocal (i.e., integral) differential equations with exterior Dirichlet boundary conditions. For the scalar SDE (1), these are nonlocal ordinary differential equations, while for a SDE system, these become nonlocal partial differential equations. The non-Gaussianity of the noise manifests as nonlocality at the level of the mean exit time and escape probability.

When we have observations on the mean exit time $u(x)$ or escape probability $P_{E}(x)$, it is thus possible to estimate $\alpha$ and other system parameters, by solving an inverse problem for the nonlocal differential equations.

It is sometimes too costly to observe system state sample paths $X_{t}$ over very long time periods [24], but is more feasible to observe (or to infer from collected data) other quantities about system evolution, such as mean exit time and escape probability. Mean residence time has been observed or measured in chemical, industrial and physiological systems [15, 26]. For example, the mean residence time for xenon in intact and surgically isolated muscles can be measured [27]. The mean residence time of xenon is longer than that predicted by a single-compartment model of gas exchange, and this leads to the understanding that a multiple-compartment model might be more accurate according to larger relative dispersion (the standard deviation of residence time divided by the mean). Escape probability has also been observed or measured in certain physical and electronic systems [11, 12, 13].

This paper is organized as follows. In section 2, we formulate our method, i.e., an inverse problem for nonlocal differential equations to estimate parameters. Numerical simulation results are presented in section 3. The paper ends with some discussions in section 4. 


\section{Methods}

A scalar symmetric $\alpha$-stable Lévy motion $L_{t}^{\alpha}$ is characterized [2, 21] by a shift coefficient which is often taken to be zero (for convenience) and a non-negative measure $\nu_{\alpha}$ defined on the state space $\mathbb{R}^{1}$ :

$$
\nu_{\alpha}(\mathrm{d} x)=C_{\alpha}|x|^{-(1+\alpha)} \mathrm{d} x
$$

with $\alpha \in(0,2)$ and $C_{\alpha}=\frac{\alpha}{2^{1-\alpha} \sqrt{\pi}} \frac{\Gamma\left(\frac{1+\alpha}{2}\right)}{\Gamma\left(1-\frac{\alpha}{2}\right)}$. For more information see [7, 32]. The generator for the solution process $X_{t}$ of (1) is ([2, p. 402]):

$$
\begin{aligned}
A \varphi= & f(\beta, x) \varphi^{\prime}(x) \\
& +\int_{\mathbb{R}^{1} \backslash\{0\}}\left[\varphi(x+y)-\varphi(x)-I_{\{|y|<1\}} y \varphi^{\prime}(x)\right] \nu_{\alpha}(\mathrm{d} y),
\end{aligned}
$$

where $I_{S}$ is the indicator function of the set $S$, i.e.,

$$
I_{S}(y)= \begin{cases}1, & \text { if } y \in S \\ 0, & \text { if } y \notin S\end{cases}
$$

We consider the mean exit time, $u(x)$, for an orbit starting at $x$, from a bounded open interval $D$. By the Dynkin formula [28, 31] for general Markov processes, as in [25, 4, 5, 14], we know that $u(x)$ satisfies the following nonlocal differential equation:

$$
\begin{aligned}
A u(x) & =-1, \quad x \in D \\
u & =0, \quad x \in D^{c},
\end{aligned}
$$

where $D^{c}=\mathbb{R}^{1} \backslash D$ is the complement of $D$.

Suppose that we have observed the mean exit time $u(x), x \in D=(a, b)$ (a small interval). We then solve the inverse problem for a nonlocal differential with exterior boundary condition (3)-(44), in order to estimate $\alpha$ and $\beta$. See [20, 3, 22] for discussions on inverse problems for partial differential equations. This is achieved by a numerical optimization

$$
\min _{\alpha, \beta} G(\alpha, \beta)
$$

where the objective function $G=\operatorname{dist}\left(u(x), u_{o b}\right)$, for an appropriate distance function 'dist' between $u$ and its observation $u_{o b}$. Note that the observation $u_{o b}$, depending on spatial location $x$, is usually in data format. To evaluate the objective function $G$ at initially guessed or approximated values of $(\alpha, \beta)$, 
we need to numerically solve (3)-(40) by a finite difference scheme in [14]. See also [8] for discussions on related simulations.

We also consider estimation of parameters using observations on escape probability for the system (11). The escape probability of a particle, starting at a point $x$, first escapes from a bounded domain $D$ and lands in a subset $E$ of $D^{c}$, is denoted by $P_{E}(x)$, and it satisfies the following nonlocal differential equation 29]

$$
\begin{aligned}
A P_{E}(x) & =0, \quad x \in D, \\
\left.P_{E}\right|_{x \in E} & =1,\left.\quad P_{E}\right|_{x \in D^{c} \backslash E}=0,
\end{aligned}
$$

where $A$ is the generator defined in (2). The subset $E$ is usually taken to be an open set ([10, Ch. 7]). We again solve the inverse problem for a nonlocal differential with exterior boundary condition (6)-(7), in order to estimate $\alpha$ and $\beta$. This is also achieved by a numerical optimization

$$
\min _{\alpha, \beta} G(\alpha, \beta),
$$

where the objective function $G=\operatorname{dist}\left(P_{E}(x), P_{E o b}\right)$, for an appropriate distance 'dist' between $P_{E}$ and its observation $P_{E o b}$. Note that the observation $P_{E o b}$, depending on spatial location $x$, is usually in data format. To evaluate the objective function $G$ at initially guessed or approximated values of $(\alpha, \beta)$, we need to numerically solve (6) -(7), also by a finite difference scheme in [14].

In both settings above, the domain $D$ can be taken as small as we like (or arbitrarily small). This offers an advantage as it uses limited amount of observational resources.

In the present paper, we only consider scalar SDEs. For SDEs in higher dimensions, both mean exit time and escape probability satisfy nonlocal partial differential equations, and our method also applies.

\section{Numerical experiments}

We now consider three examples to illustrate our method for estimating parameters in stochastic dynamical systems with non-Gaussian Lévy noise. For numerical optimization, we use Matlab's built-in function fminbnd, which is a hybrid scheme, using both successive parabolic interpolation and golden section search to find a minimizer for an objective function on a fixed interval. In the examples below, we use simulated data for $u_{o b}$ and $P_{E o b}$, in order to illustrate our method. Our following numerical experiments are reproducible by Matlab coding the finite difference scheme in [14], together with the Matlab's built-in function fminbnd for numerical optimization. 
Example 1. Consider a scalar Ornstein-Uhlenbeck system

$$
d X_{t}=-X_{t} d t+d L_{t}^{\alpha}, X_{0}=x
$$

In this example, $f(x)=-x$. Suppose that we have observed the mean residence time $u_{o b}(x)$ for $x \in D=(-2,2)$ and $(-0.1,0.1)$. Let us find out estimation of $\alpha$ by solving the inverse problem of the following nonlocal differential equation:

$$
\begin{aligned}
A u(x) & =-1, \quad x \in D \\
u & =0, \quad x \in D^{c},
\end{aligned}
$$

where the generator $A$ is

$$
\begin{aligned}
A u= & -x u^{\prime}(x) \\
& +\int_{\mathbb{R}^{1} \backslash\{0\}}\left[u(x+y)-u(x)-I_{\{|y|<1\}} y u^{\prime}(x)\right] \nu_{\alpha}(\mathrm{d} y),
\end{aligned}
$$

and $D^{c}=\mathbb{R}^{1} \backslash D$ is the complement set of $D$.

Using the $L^{2}$ norm, we define an objective function

$$
G(\alpha)=\frac{\left\|u(\alpha, x)-u_{o b}(x)\right\|_{2}^{2}}{\left\|u_{o b}(x)\right\|_{2}^{2}}
$$

and the estimation of $\alpha \in(0,2)$ is taken to be the minimizer, i.e.,

$$
\alpha_{E}=\arg \min _{\alpha} G(\alpha) .
$$

To test our method, we generate the observation $u_{o b}(x)$ by numerically solving (10) with $\alpha=0.6$. The values of $u(\alpha, x)$ are from finite difference [14] discretization of (10) with $\alpha$ as a parameter to be determined. Figure 1 shows accurate estimation of $\alpha$ on a smaller domain $D=(-0.1,0.1)$, as well as on a larger domain $D=(-2,2)$.

Example 2. Consider

$$
d X_{t}=\left(X_{t}-X_{t}^{3}\right) d t+d L_{t}^{\alpha}, X_{0}=x
$$

$f(x)=x-x^{3}$.

We estimate $\alpha$, using observation on escape probability $P_{E o b}$. Namely, we solve an inverse problem for the following nonlocal differential equation

$$
\begin{aligned}
A P_{E}(x) & =0, \quad x \in D \\
\left.P_{E}\right|_{x \in E} & =1,\left.\quad P_{E}\right|_{x \in D^{c} \backslash E}=0,
\end{aligned}
$$



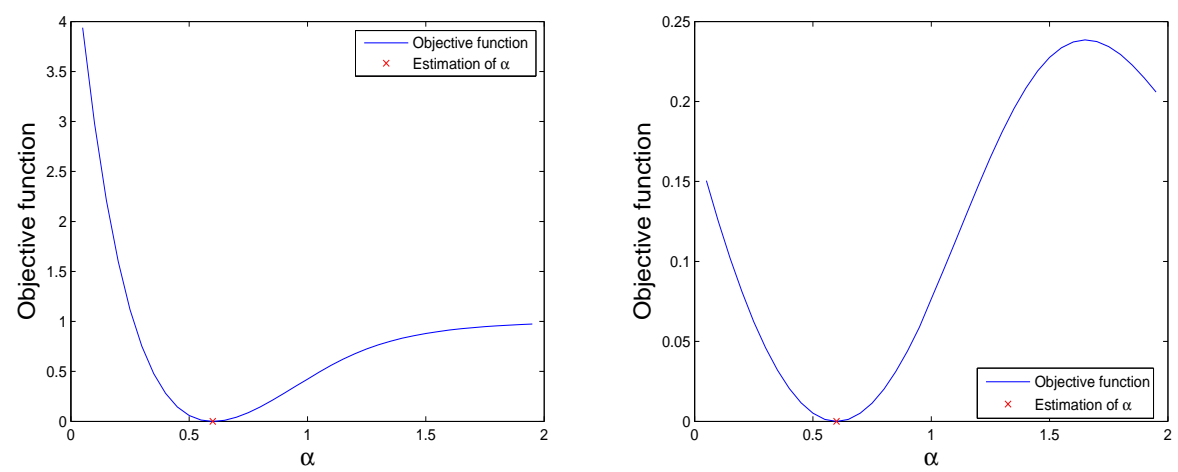

Figure 1: Estimation of $\alpha$ on domains $D=(-0.1,0.1)$ (left) and $D=(-2,2)$ (right) with observation on mean exit time: True value $\alpha=0.6$.
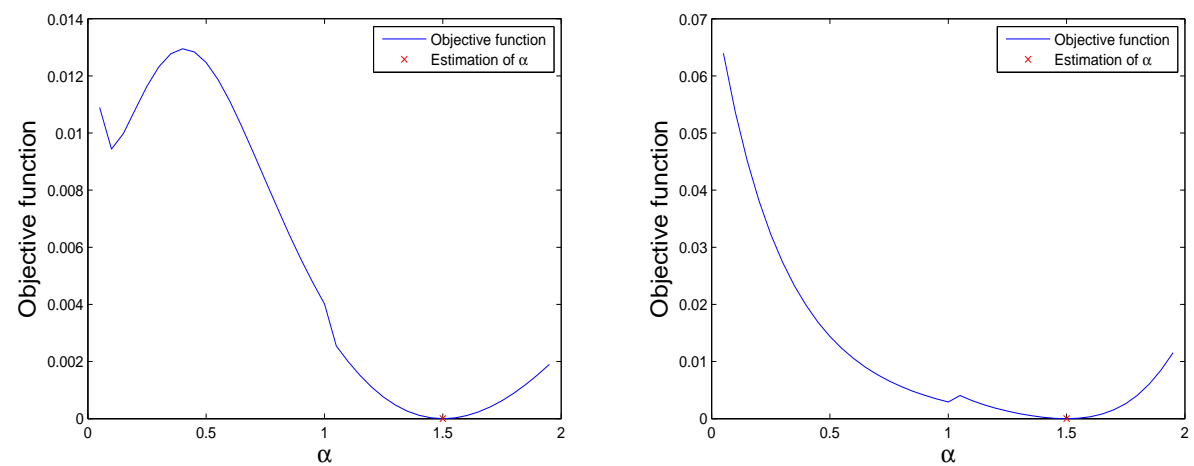

Figure 2: Estimation of $\alpha$ on domains $D=(-0.1,0.1)$ (left) and $D=(-2,2)$ (right) using observation on escape probability: True value $\alpha=1.5$.

where $A$ is the generator defined in (2). Defining an objective function

$$
G(\alpha)=\frac{\left\|P_{E}(\alpha, x)-P_{E o b}(x)\right\|_{2}^{2}}{\left\|P_{E o b}(x)\right\|_{2}^{2}}
$$

the estimation of $\alpha$ is $\alpha_{E}=\arg \min _{\alpha} G(\alpha)$. To test our method, we generate the observation $P_{E o b}(x)$ by numerically solving (13)-(14) with $\alpha=1.5$. The values of $P_{E}(\alpha, x)$ are from finite difference [14 discretization of (13)-(14) with $\alpha$ as a parameter to be determined. Figure 0 shows the estimation of $\alpha=1.5$ on two different domains.

Example 3. Consider

$$
d X_{t}=\left(X_{t}-\beta X_{t}^{3}\right) d t+d L_{t}^{\alpha}, X_{0}=x .
$$




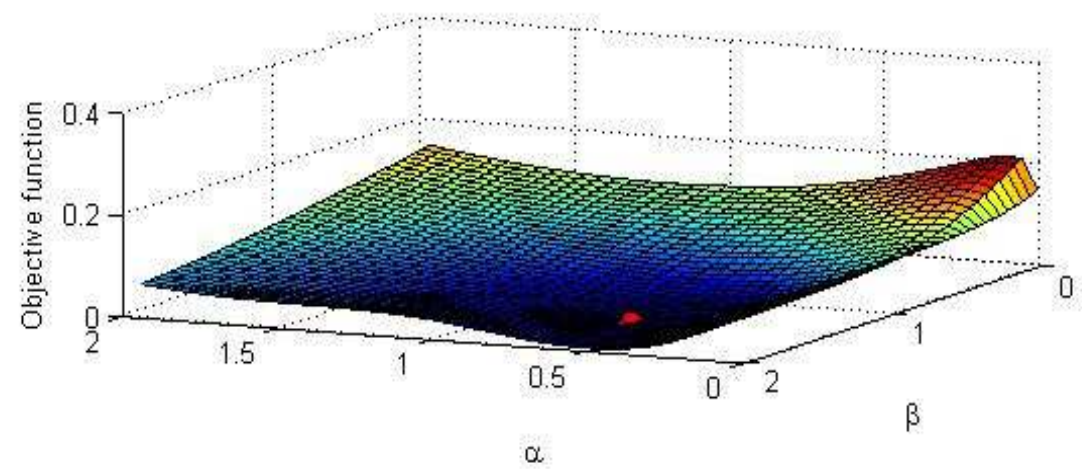

Figure 3: Estimation of $\alpha$ and $\beta$ by observing mean exit time with true value of $\alpha=0.6$ and true value of $\beta=1.5$. The estimated $\alpha$ is 0.59858 and the estimated $\beta$ is 1.51 .

$f(x)=x-\beta x^{3}$ where $\beta$ is a positive parameter .

In this example, we use observations of either mean exit time or escape probability to estimate unknown parameters. Let the observation of mean exit time be $u_{o b}$ and the observation of escape probability be $P_{E o b}$. Defining an objective function

$$
G_{1}(\alpha, \beta)=\frac{\left\|u(x, \alpha, \beta)-u_{o b}(x)\right\|_{2}^{2}}{\left\|u_{o b}(x)\right\|_{2}^{2}},
$$

and

$$
G_{2}(\alpha, \beta)=\frac{\left\|P_{E}(x, \alpha, \beta)-P_{E o b}(x)\right\|_{2}^{2}}{\left\|P_{E o b}(x)\right\|_{2}^{2}},
$$

respectively, we obtain estimations of parameters by minimizing these functions separately. Results are shown in Figure 3 for using observation of mean exit time and Figure 4 for using observation of escape probability.

\section{Discussions}

In summary, we have devised a method to estimate the non-Gaussianity parameter $\alpha$, and other system parameters, for non-Gaussian stochastic dynamical systems, using observations on either mean exit time or escape probability. It is based on solving an inverse problem for a deterministic, nonlocal partial differential equation via numerical optimization.

When the noise has a Gaussian component modeled by a Brownian motion $B_{t}$, the generator $A$ in nonlocal partial differential equations (3) and (6) contains an extra Laplacian term $\Delta u$ and our method still works. Especially, 


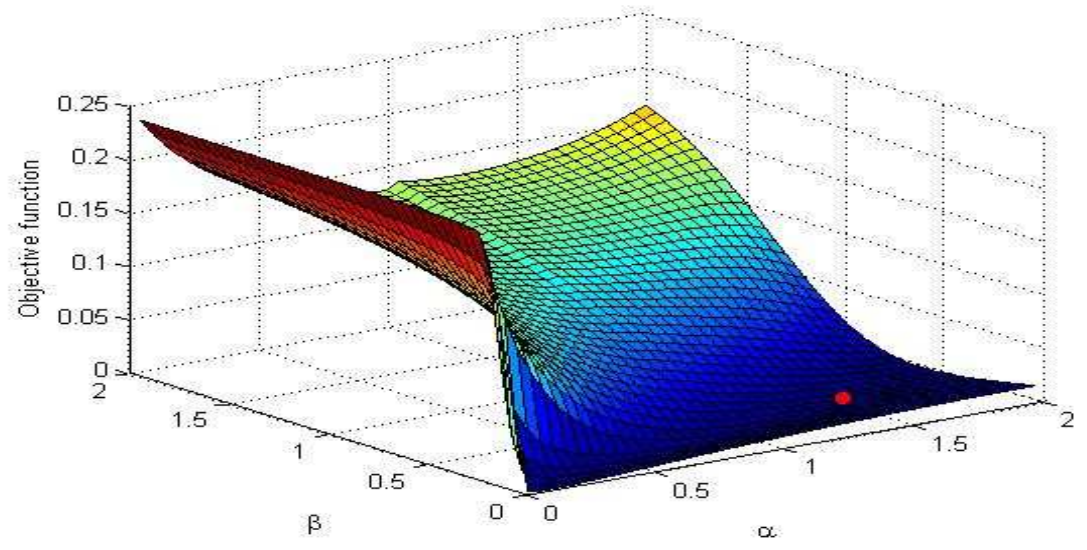

Figure 4: Estimation of $\alpha$ and $\beta$ by observing escape probability with true value of $\alpha=1.5$ and true value of $\beta=0.4$. The estimated $\alpha$ is 1.5288 and the estimated $\beta$ is 0.401 .

if the noise has only Gaussian component, the generator $A$ is $\Delta u$ (and the nonlocal term is absent) and our method remains valid.

The existing methods for estimating the non-Gaussianity parameter $\alpha$ require observations on system state sample paths for long time periods or probability densities on very large spatial domain. The method proposed here, instead, requires observations on either mean exit time or escape probability only for an arbitrarily small spatial domain. This new method is especially beneficial for systems where either mean exit time or escape probability is relatively easy to observe.

Acknowledgement. Part of this work was done while authors were at the Institute for Pure and Applied Mathematics (IPAM), Los Angeles, USA. This work was partly supported by the NSF Grant 1025422. We thank Xiaofan Li for help with numerical discretization of nonlocal partial differential equations and Mike McCourt for help with numerical optimization.

\section{References}

[1] R. J. Adler, R. E. Feldman and M. S. Taqqu (eds.), A Practical Guide to Heavy Tails. Birkhauser, Berlin, 1998.

[2] D. Applebaum, Lévy Processes and Stochastic Calculus. Cambridge University Press, Cambridge, UK, 2004. 
[3] Yu. Ya Belov, Inverse Problems for Partial Differential Equations. VSP, 2002 ,

[4] J. Brannan, J. Duan and V. Ervin, Escape Probability, Mean Residence Time and Geophysical Fluid Particle Dynamics, Physica D 133 (1999), 23-33.

[5] J. Brannan, J. Duan and V. Ervin, Escape probability and mean residence time in random flows with unsteady drift. Mathematical Problems in Engineering Volume 7 (2001), Issue 1, Pages 55-65. doi:10.1155/S1024123X01001521

[6] D. Brockmann, Human Mobility and Spatial Disease Dynamics. Chapter in Reviews of Nonlinear Dynamics and Complexity, H. G. Schuster (ed.), Wiley-VCH, 2009.

[7] Z. Chen, P. Kim and R. Song, Heat kernel estimates for Dirichlet fractional Laplacian. J. European Math. Soc. 12 (2010), 1307-1329.

[8] W. Dai, On the conflict of truncated random variable vs. heavy-tail and long range dependence in computer and network simulation, Journal of Computational Information Systems 7:5 (2011) 1488-1499.

[9] P. D. Ditlevsen, Observation of $\alpha$-stable noise induced millennial climate changes from an ice record. Geophys. Res. Lett. 26 (1999), 14411444 .

[10] J. Duan, An Introduction to Stochastic Dynamics. Cambridge University Press, New York, 2015.

[11] H. Ebel, R. Svagera, W. S.M. Werner and M. F. Ebel, Escape Probability of Electrons in Total Electron yield experiments. JCPDS International Centre for Diffraction Data, Vol 41, p.367-378, 1999.

[12] S. A. Elwakil, E. A. Saad, M. T. Attia and S. K. El-Labany, Particle escape probability from spherical geometry. Astrophysics and Space Science Volume 150, Number 1 (1988), 9-15.

[13] R. G. Forbes, On the need for a tunneling pre-factor in FowlerNordheim tunneling theory. Journal of Applied Physics 103 (11)(2008): 114911.

[14] T. Gao, J. Duan, X. Li and R. Song, Mean exit time and escape probability for dynamical systems driven by Lévy noise. SIAM J. Sci. Computing Vol. 36, No. 3, pp. A887-A906, 2014. 
[15] F. Ghirelli and B. Leckner, Transport equation for the local residence time of a fluid. Chemical Engineering Science 59 (2004) 513-523.

[16] Y. Hu and H. Long, Parameter estimation for Ornstein-Uhlenbeck processes driven by $\alpha$-stable Lévy motions. Communications on Stochastic Analysis, 2007, 1: 175-192.

[17] Y. Hu and H. Long, Least squares estimator for Ornstein-Uhlenbeck processes driven by $\alpha$-stable motions. Stochastic Process Appl, 2009, 119: $2465-2480$.

[18] N. E. Humphries, H. Weimerskirch, N. Queiroz, E. J. Southall and D. W. Sims, Foraging success of biological Lévy flights recorded in situ, Proc. Natl. Acad. Sci. 109(19):7169-7174 (2012)

[19] I. A. Ibragimov and R. Z. Has'minskii, Statistical EstimationAsymptotic Theory. Springer, New York, 1981.

[20] V. Isakov, Inverse Problems for Partial Differential Equations. Springer, New York, 1998.

[21] A. Janicki and A. Weron, Simulation and Chaotic Behavior of $\alpha$-Stable Stochastic Processes, Marcel Dekker, Inc., 1994.

[22] A. Kirsch, An Introduction to the Mathematical Theory of Inverse Problems. Springer, New York, 1996.

[23] H. Long and L. Qian, Nadaraya-Watson estimator for stochastic processes driven by stable Lévy motions. Electronic Journal of Statistics, Vol. 7 (2013) 13871418

[24] F. Moss and P. V. E. McClintock (eds.), Noise in Nonlinear Dynamical Systems. Volume 1: Theory of Continuous Fokker-Planck Systems (2007); Volume 2: Theory of Noise Induced Processes in Special Applications (2009); Volume 3: Experiments and Simulations (2009). Cambridge University Press.

[25] T. Naeh, M. M. Klosek, B. J. Matkowsky and Z. Schuss, A direct approach to the exit problem, SIAM J. Appl. Math. 50 (1990), 595-627.

[26] E. B. Nauman, Residence Time Distributions. In Handbook of Industrial Mixing: Science and Practice. Wiley Interscience, pp. 117 (2004).

[27] J. A. Novotny, E. C. Parker, S. S. Sruvanshi, G. W. Albin and L. D. Homer, Contribution of tissue lipid to long xenon residence times in muscle. J Appl Physiol. 1993 May;74(5):2127-34. 
[28] B. Oksendal, Applied Stochastic Control Of Jump Diffusions. SpringerVerlag, New York, 2005.

[29] H. Qiao, X. Kan and J. Duan, Escape probability for stochastic dynamical systems with jumps. Springer Proceedings in Mathematics 8 Statistics, Vol. 34, p. 195-216, 2013.

[30] G. Samorodnitsky and M. S. Taqqu, Stable Non-Gaussian Random Processes, Chapman and Hall, 1994.

[31] K.-I. Sato, Lévy Processes and Infinitely Divisible Distributions, Cambridge University Press, Cambridge, 1999.

[32] D. Schertzer, M. Larcheveque, J. Duan, V. Yanovsky and S. Lovejoy, Fractional Fokker-Planck equation for nonlinear stochastic differential equations driven by non-Gaussian Lévy stable noises. J. Math. Phys., 42 (2001), 200-212.

[33] M. F. Shlesinger, G. M. Zaslavsky and U. Frisch, Lévy Flights and Related Topics in Physics (Lecture Notes in Physics, 450. Springer-Verlag, Berlin, 1995).

[34] T. H. Solomon, E. R. Weeks, and H. L. Swinney, Observation of anomalous diffusion and Lévy flights in a two-dimensional rotating flow. Phys. Rev. Lett. 71, 3975 - 3978 (1993).

[35] W. A. Woyczynski, Lévy processes in the physical sciences. In Lévy Processes: Theory and Applications, O. E. Barndorff-Nielsen, T. Mikosch and S. I. Resnick (Eds.), 241-266, Birkhäuser, Boston, 2001.

[36] J. Yang and J. Duan, Quantifying model uncertainties in complex systems. Progress in Probability, Vol. 65, p.49-80, 2011. 